medRxiv preprint doi: https://doi.org/10.1101/2021.10.25.21265372; this version posted October $26,2021$. The copyright holder for this preprint (which was not certified by peer review) is the author/funder, who has granted medRxiv a license to display the preprint in It is made available under a CC-BY-NC-ND 4.0 International license .

Title

The preoperative abundance of Prevotella associates with anastomotic healing after colorectal surgery

\title{
Authors list
}

Konstantina Zafeiropoulou1,2, Emmeline G. Peters1,3, Daan J. Brinkman1,3, Andrew Y.F. Li Yim¹,4, Boudewijn J.J. Smeets ${ }^{3}$, Theodorus B.M. Hakvoort ${ }^{1}$, Evgeni Levin5,6, Mark Davids 5 , Misha D.P. Luyer ${ }^{3}$, Wouter J. de Jonge ${ }^{1,7,8,{ }^{*}}$, Joep P.M. Derikx²,8

\section{Affiliations}

1Tytgat Institute for Liver and Intestinal Research, Amsterdam Gastroenterology Endocrinology and Metabolism, Amsterdam University Medical Centers, University of Amsterdam, 1105 BK Amsterdam, The Netherlands

2Department of Pediatric Surgery, Emma Children's Hospital, Amsterdam University Medical Centers, University of Amsterdam, 1105 AZ Amsterdam, The Netherlands

3Department of Surgery, Catharina Hospital, 5623 EJ Eindhoven, The Netherlands

4Department of Clinical Genetics, Genome Diagnostics Laboratory, Amsterdam Reproduction and Development, Amsterdam University Medical Centers, University of Amsterdam, 1105 AZ Amsterdam, The Netherlands

5Laboratory of Experimental Vascular Medicine, Amsterdam University Medical Centers, University of Amsterdam, 1105 AZ Amsterdam, The Netherlands

6Horaizon BV, 2625 GZ Delft, The Netherlands

7Department of General, Visceral, Thoracic and Vascular Surgery, University Hospital Bonn, 53127 Bonn, Germany

8Shared senior authorship

*Correspondence: w.j.dejonge@amsterdamumc.nl 
medRxiv preprint doi: https://doi.org/10.1101/2021.10.25.21265372; this version posted October 26, 2021. The copyright holder for this preprint (which was not certified by peer review) is the author/funder, who has granted medRxiv a license to display the preprint in It is made available under a CC-BY-NC-ND 4.0 International license

\section{Summary}

Anastomotic leakage (AL) is a frequent and potentially life-threatening postoperative complication in patients undergoing colorectal surgery with a multifactorial yet unknown pathophysiology. In the present study, we aimed to unravel AL etiology and intestinal anastomotic healing $(\mathrm{AH})$ physiology focusing on both host epithelial and gut microbial components. We make use of patients-related samples and two high throughput next generation sequencing analysis approaches, including anastomotic tissue-associated bulk RNA sequencing and preoperative fecal 16S rRNA gene sequencing. We find that in the absence of host epithelial gene expression differences, the preoperative fecal microbiota of patients who undergo colorectal resection with successful AH differs in the group of patients who develop AL in the abundance of Prevotella and suggests a potential beneficial role of Prevotella that may prove useful as predictive biomarker for $\mathrm{AH}$. 
medRxiv preprint doi: https://doi.org/10.1101/2021.10.25.21265372; this version posted October $26,2021$. The copyright holder for this preprint (which was not certified by peer review) is the author/funder, who has granted medRxiv a license to display the preprint in It is made available under a CC-BY-NC-ND 4.0 International license .

Anastomotic leakage (AL) is a severe postoperative complication in patients undergoing colorectal surgery, which negatively impacts hospital costs, morbidity, mortality, re-operation rates, and duration of hospitalization (Hammond et al., 2014). Although immunosuppressive medication and distal location of the anastomosis are known risk factors for AL (Sahami et al., 2016), its pathophysiology remains poorly understood and options to prevent $A L$ are limited. The unpredictability of $A L$ can be attributed to a lack of fundamental understanding of the processes underlying anastomotic healing (AH) (Bosmans et al., 2015). The current hypothesis suggests a multifactorial etiology, with host genetic make-up, perioperative inflammation and intestinal microbiota being key players (Lee et al., 2018).

Previous work from our group has demonstrated an association between $A L$ and postoperative ileus (POI) (Peters et al., 2017). Although POI-associated stasis of the luminal content and preoperative microbiome signal is now profound (Shogan et al., 2020), the role of gut microbiota in AL remains unclear. Earlier studies point to low abundance of collagenase-producing strains (i.e. Enterococcus faecalis) that may predominate in the lumen of patients with colorectal cancer (CRCA) and drive AL via collagen breakdown (i.e., matrix metalloproteinase 9) (Shogan et al., 2015). Further findings show a predictive value of the anastomotic tissue-related mucus microbiome in the absence of C-seal treatment, characterized by decreased microbial diversity and increased relative abundance of mucin-degrading commensal members (van Praagh et al., 2019). However, there is currently no consensus on AL-associated gut microbial dysbiosis. Respectively, findings from the latter cohort indicate pre-existing differences in the gene expression, with the collagen cross linking pathway being downregulated (van Praagh et al., 2021), but that needs to get confirmed in larger cohorts and mechanistically challenged.

In the present study, we explored the AL pathogenesis in a stepwise approach using two highthroughput next generation sequencing techniques (i.e., tissue RNA sequencing and fecal 16S rRNA gene sequencing). Patients with CRCA who underwent colorectal resection were herein included; all patients were enrolled in a multicenter double-blind randomized controlled study from August 2014 until February 2017 (SANICS II trial) (Peters et al., 2018).

We initially interrogated the anastomotic tissue-associated transcriptome at time of surgery. Samples were obtained from a SANICS II-subgroup of 18 patients who developed AL after colorectal surgery, and 18 age, sex and body mass index (BMI)-matched patients with successful postoperative AH (Table 1). A pipeline of pre-processing steps was applied, including quality control of the RNA sequencing raw reads (Andrews, 2010; Ewels et al., 2016), alignment to the human genome (Dobin et al., 2013), annotation (Aken et al., 2017), post-alignment processing analysis (Li et al., 2009) and reads counting (Liao et al., 2013). Unsupervised exploratory analyses suggested no clear separation between AL and AH through hierarchical clustering analysis for the 150 most variable genes (Figure S1A). Subsequent supervised analyses (Robinson et al., 2010) identified no difference in expression observed between the $A L$ and $A H$ patient cohorts either (Figure S1B). This finding highlighted further our need to address the role of gut microbiota in the process of $\mathrm{AL}$, rather than pre-existing differences in host gene expression at the time of surgery.

Therefore, we included a SANICS II-subgroup of 57 patients (13 women) who participated in the preoperative fecal microbiota study providing a fecal sample one day prior to the colorectal surgery; 11 out 
medRxiv preprint doi: https://doi.org/10.1101/2021.10.25.21265372; this version posted October $26,2021$. The copyright holder for this preprint (which was not certified by peer review) is the author/funder, who has granted medRxiv a license to display the preprint in It is made available under a CC-BY-NC-ND 4.0 International license .

of the 57 patients developed $\mathrm{AL}$, while the remaining 46 did not (AH). AL was diagnosed based on radiologic imaging (5 out of $11 \mathrm{AL}$ ) or confirmed during re-operation (6 out $11 \mathrm{AL}$ ) and treated with antibiotics (2 out of 11), drainage ( 3 out of $11 \mathrm{AL}$ ), secondary surgery (5 out of $11 \mathrm{AL}$ ) or untreated in the absence of symptoms (1 out of $11 \mathrm{AL}$, Table 1). There was no association between AL development and resection's location (46 colonic; 11 rectal resections), neoadjuvant therapy (chemo-radiotherapy in 3 out of $11 \mathrm{AL}$ ) or performance of colostomy ( 9 out of 57 patients); no difference in surgery duration between AL and AH was found either (Table 1). Thirty-three patients underwent a laparoscopy and 16 an open resection, while eight patients were converted to open surgery, which tended to be significantly associated with the development of $\operatorname{AL}\left(X^{2}(2, N=57)=5.936, P=0.051\right.$, Table 1$)$. There was no difference in age, sex nor BMI between the AL and AH patients, whilst no association was found between smoking behavior and postoperative diagnosis of AL. All 57 patients were classified up to American society of anesthesiologists (ASA) class III; two out of the 57 were treated with non-steroidal anti-inflammatory drugs (NSAID) before surgery, with no association between the ASA score or NSAID treatment and AL retrieved $(P>0.05$ for all aforementioned analyses, Table 1).

We profiled the preoperative fecal microbiota structure of all 57 patients using 16R rRNA gene sequencing targeting the V3-V4 regions (Kozich et al., 2013). A pipeline of pre-processing steps was herein conducted as well, including reads truncation, merging, amplicon sequence inference (Edgar, 2016), and annotation using the SILVA 16S ribosomal database 132 (Quast et al., 2013); amplicon sequence variants (ASV) complete count table was stored as a Phyloseq object (McMurdie and Holmes, 2013). We found no difference in fecal $\alpha$ diversity between $\mathrm{AL}$ and $\mathrm{AH}$ groups, arguing against a profound dysbiosis pre-existing preoperatively (Figure 1A). AL development could not explain the variation in fecal ASV community structure (explored using the vegan package (Oksanen J., 2020), Figure 1B), which was further confirmed by the local contribution to $\beta$ diversity (LCBD) analysis where no difference was observed between the two groups (Figure 1A). Using the Bioenv workflow, a subset of ASVs belonging to 21 genera was suggested as major determinants of the community complexity, explaining $83.5 \%$ of the variance described by the complete ASV dataset (Table S1).

We then looked at the preoperative fecal microbial composition pursuing differential analysis using the DESeq2 package (Love et al., 2014) in taxa relative abundance between patients who developed AL after surgery and those who did not at both ASVs and agglomerated at genus levels. All 57 patients were characterized by a total of 2,577 distinct, non-mitochondrial ASVs. One hundred and six ASVs out of the 2,577 (3.9\%) differed significantly between the two groups, with 90 and 16 being significantly increased and decreased in feces of AH patients, respectively (Table S2). At genus level, 16 taxa differed significantly between $A L$ and $A H$, with only two being significantly increased in patients who developed AL after surgery (P (adjusted) $<0.05$, Figure $1 \mathrm{C}$ and Table S2). The remaining 14 displayed significantly increased preoperative relative abundance in the feces of $\mathrm{AH}$ group, suggesting a potential beneficial role in intestinal $\mathrm{AH}$.

To challenge the meaning of these significant preoperative fecal microbial composition differences in the development of $\mathrm{AL}$ and physiology of $\mathrm{AH}$, we explored potential correlations between their relative abundances, patient-related individual factors (i.e., age, BMI) and preoperative serum concentrations of the 
medRxiv preprint doi: https://doi.org/10.1101/2021.10.25.21265372; this version posted October $26,2021$. The copyright holder for this preprint (which was not certified by peer review) is the author/funder, who has granted medRxiv a license to display the preprint in It is made available under a CC-BY-NC-ND 4.0 International license .

TNF- $\alpha$, interleukin $1 \beta$ (IL-1 $\beta$ ), IL-6, IL-8, IL-10 and IL-12 cytokines. Of the 16 discriminatory genera, only the relative abundance of Paraprevotella correlated significantly negatively with the preoperative serum concentration of the cytokine IL-12, suggesting that the remaining 15 were less likely to be explained by differences in the inflammatory status between the two groups, but rather $\mathrm{AL}$ - and $\mathrm{AH}$ - specific (Figure S2).

Prevotella 2, Prevotella 9, Alloprevotella, Paraprevotella and unclassified Prevotellaceae, five out of the 14 (35.7\%) discriminatory taxa with significantly increased relative abundance in the AH group, are all amongst the essential components of the Prevotella enterotype (highlighted with dark green in the bar plot in Figure 1C). In the present cohort, Prevotella 9 was also listed among the 21 Bioenv-selected taxa found to explain $83.5 \%$ of the fecal microbial variance (indicated with asterisk in Figure 1C, Table S1), signifying its importance in the microbiome structure of the 57 patients. Therefore, we proceeded one step further and performed statistical analysis at enterotypes level (Arumugam et al., 2011) that highlighted the beneficial effect of preoperative Prevotella when no subjects with a predicted Prevotella enterotype developed AL (X2 $(2, N=57)=7.658, P=0.10$, Figure 1D).

Our data suggest a beneficial role of Prevotella in AH. We confirmed it further with the means of feature importance analysis. Using the complete count table aggregated at genus level, conditional random forest classifier (Hothorn et al., 2006) distinguished patients who developed AL from patients who did not with an "out-of-bag" error rate of $19.3 \%$. This was significantly more effective than random classification (permutation analysis of variance $P=0.019$ and area under the curve $=0.660)$. Importantly, genera Prevotella 9 and Prevotella 7 were among the most influential taxa, with conditional variance importance suggesting them as the third (variance importance $=4 \mathrm{E}-04$ ) and twelfth (variance importance $=2 \mathrm{E}-04$ ) most prominent genera respectively (indicated with stripes at Figure 1C).

Overall, we demonstrated that in the absence of host gene expression differences, the gut microbiota of patients who undergo colorectal resection with successful AH differs in the group of patients who develop AL in the abundance of Prevotella. We used an elaborative pipeline of statistical analyses that enables us to suggest that the Prevotella-related microbial signals in the group of patients who do not develop AL may prove useful as predictive biomarkers for $\mathrm{AH}$. Prevotella enterotype has been distinctly described in loose feces, as defined by increased Bristol Stool Scale ratings of their stool consistency, a proxy for the intestinal colon transit time (Vandeputte et al., 2016). The abundance of Prevotella enterotype in subjects with loose feces, may render easier gastrointestinal passage resulting in less stasis and luminal distention and thus better anastomotic healing, a functional partial explanation for the present findings. We speculate that passage of harder stools will hinder an anastomosis from healing.

Decreased Prevotella has been also described in AL mucus-associated microbiome, when bacterial DNA was isolated from "donuts" of patients in whom a stapled colorectal anastomosis was made, yet that was not significant when the non C-seal and C-seal covered anastomosis patients were analyzed together (van Praagh et al., 2019). In following up analyses, we wish to validate a Prevotella-associated predictive algorithm for $\mathrm{AH}$ in a separate cohort of patients (i.e., REVEAL cohort (Jongen et al., 2016)). We believe that this can have a significant clinical and economic impact once implemented in CRCA surgeries, where the surgeon has options to prevent surgical anastomoses in patients at risk for developing AL. 
medRxiv preprint doi: https://doi.org/10.1101/2021.10.25.21265372; this version posted October 26, 2021. The copyright holder for this preprint (which was not certified by peer review) is the author/funder, who has granted medRxiv a license to display the preprint in It is made available under a CC-BY-NC-ND 4.0 International license.

\section{Acknowledgements}

The authors would like to thank all SANICS II trial participants. J.D. and W.J are funded by the Dutch Research Council (ZonMW-NWO) research program Vidi project, No. 91719343 and AMC-stipendium 200402.

\section{Authors Contributions}

Conceptualization, E.P., W.J. and M.L.; Data curation, E.P.; Formal analysis. K.Z., A.L.Y. and M.D., Funding acquisition: W.J., J.D. and M.L.; Investigation: E.P., D.B., B.S. and T.H.; Project administration: E.P.; Resources: W.J.; Supervision: E.L., J.D., M.L. and W.J.; Visualization: K.Z. and A.L.Y.; Writing - original draft: K.Z.; Writing - review \& editing: K.Z., J.D. and W.J.

\section{Declaration of Interests}

The authors declare that the research was conducted in the absence of any commercial or financial relationships that could be construed as a potential conflict of interest.

\section{Data and Code Availability}

All data produced in the present study are available upon reasonable request to the authors.

\section{Additional Resources}

The study was registered at ClinicalTrials.gov (NCT02175979, https://clinicaltrials.gov/ct2/show/NCT021759 79) and trialregister.nl (NTR4670, https://www.trialregister.nl/trial/4494).

\section{Ethical Considerations}

The study was approved by the Medical Ethics Committee of the Catharina Hospital. Informed consent was obtained from all subjects. 
medRxiv preprint doi: https://doi.org/10.1101/2021.10.25.21265372; this version posted October $26,2021$. The copyright holder for this preprint (which was not certified by peer review) is the author/funder, who has granted medRxiv a license to display the preprint in It is made available under a CC-BY-NC-ND 4.0 International license

Table \& Figure legends

Table 1. Patient Characteristics

Table S1. Bioenv selected taxa explain $\mathbf{8 3 . 5 \%}$ of the variance in fecal microbiota structure of preoperative patients described by the complete ASV dataset agglomerated at genus level.

Table S2. Taxa with significantly different relative abundance in preoperative fecal samples of $A L$ and $A H$ patients; DESeq2 analysis at ASV and genus level.

Figure 1. Preoperative abundance of Prevotella associates with AH after colorectal surgery in the absence of profound preoperative AL-related fecal microbial dysbiosis. (A) Alpha diversity indices (i.e., Richness, Shannon, Simpson diversity) and local contribution to beta diversity (LBCD) of preoperative fecal samples (rarefied to 30,000 reads) from patients who underwent colorectal surgery $(n=57)$; (B) Nonmetric multidimensional scaling plots of the fecal beta diversity based on unweighted UniFrac distance (upper panel) and Bray-Curtis dissimilarity index (lower panel); (C) Log2fold changes of the 16 discriminatory between AL and $\mathrm{AH}$ patients genera ( $\mathrm{P}$ (adjusted) < 0.05, see also Table S2 and Figure S2); asterisk indicates Bioenv-selected taxa suggested as major determinants of the community complexity (see also Table S1); stripes indicate influential taxa based on the conventional random forest classification-feature importance analysis; taxa essential components of the Prevotella enterotype are indicated with dark green (same color as Prevotella enterotype in panel (D)); the red dashed arrows highlight the significant absence of Prevotella-related taxa in $A L$; (D) Relative contribution of the three enterotypes in the $A L$ and $A H$ patients.

Figure S1. No difference found in the colonic tissue transcriptional profile of patients who undergo colorectal surgery and develop AL. (A) Volcano plot of genes in AL compared to AH patients indicates the absence of significant DEG at surgery. (B) Heatmap representation of the hierarchical clustering analysis of the 150 most variable gene suggests no clear clustering between $\mathrm{AL}$ and $\mathrm{AH}$ patients.

Figure S2. Lack of significant correlation between the microbial discriminatory and preoperative individual components suggests their AL-specific nature. Kendall rank correlations between the relative abundance of the 16 discriminative genera, participants characteristics and preoperative serum cytokines concentrations; Benjamini-Hochberg correction was applied for multiple testing. 
medRxiv preprint doi: https://doi.org/10.1101/2021.10.25.21265372; this version posted October $26,2021$. The copyright holder for this preprint (which was not certified by peer review) is the author/funder, who has granted medRxiv a license to display the preprint in It is made available under a CC-BY-NC-ND 4.0 International license

Table 1. Patient Characteristics

RNA sequencing analysis $(n=36)$

\begin{tabular}{|c|c|c|}
\hline & $A L(18)$ & $\mathrm{AH}(18)$ \\
\hline CRCA, n (\%) & $18(100)$ & $18(100)$ \\
\hline Age (y) & $68.8(1.7)$ & $67.8(2.5)$ \\
\hline Sex, M/F & $15 / 3$ & $15 / 3$ \\
\hline BMI $\left(\mathrm{kg} / \mathrm{m}^{2}\right)$ & $25.9(0.7)$ & $26.5(0.86)$ \\
\hline \multicolumn{3}{|l|}{ Fecal microbiota analysis $(n=57)$} \\
\hline & $A L(11)$ & $\mathrm{AH}(46)$ \\
\hline Age (y) & $67.5(3.20)$ & $66.8(1.44)$ \\
\hline Sex, M/F & $8 / 3$ & $36 / 10$ \\
\hline $\mathrm{BMI}\left(\mathrm{kg} / \mathrm{m}^{2}\right)$ & $27.5(0.94)$ & $27.2(0.55)$ \\
\hline Smoking, $\mathrm{n}(\%)$ & $1(9.1)$ & $6(13.0)$ \\
\hline NSAID, n (\%) & 0 & $2(4.4)$ \\
\hline ASA class I, n (\%) & 0 & $7(15.2)$ \\
\hline ASA class II, n (\%) & $8(72.7)$ & $31(67.4)$ \\
\hline ASA class III, n (\%) & $3(27.3)$ & $8(17.4)$ \\
\hline CRCA, n (\%) & $10(90.9)$ & $42(91.3)^{a}$ \\
\hline Neoadjuvant therapy, RT/CRT* & $0 / 3$ & $1 / 6$ \\
\hline Resection, colon/rectum & $8 / 3$ & $38 / 8$ \\
\hline Laparoscopic resection, n (\%) & $4(36.4)$ & $29(63.0)$ \\
\hline Conversion to open surgery, n (\%) & $4(36.4)$ & $4(8.7)$ \\
\hline Open surgery, n (\%) & $3(27.3)$ & $13(28.3)$ \\
\hline Surgery duration (min) & $183.4(15.1)$ & $187.7(10.3)$ \\
\hline Colostomy during surgery, n (\%) & $2(18.2)$ & $8(17.4)$ \\
\hline AL diagnosis with radiologic imaging, $\mathrm{n}(\%)$ & $5(45.5)$ & N/A \\
\hline AL diagnosis confirmed with re-operation, $\mathrm{n}(\%)$ & $6(54.5)$ & N/A \\
\hline AL asymptomatic, $\mathrm{n}(\%)$ & $1(9.1)$ & N/A \\
\hline AL treatment with antibiotics, $\mathrm{n}(\%)$ & $2(18.2)$ & N/A \\
\hline AL treatment with drainage, $\mathrm{n}(\%)$ & $3(27.3)$ & N/A \\
\hline AL treatment with surgery, $\mathrm{n}(\%)$ & $5(45.5)$ & N/A \\
\hline
\end{tabular}

Values are mean (SEM) unless otherwise defined. In categorical variables, percentage (\%) refers to the $A L$ or $\mathrm{AH}$ grouping. Independent samples t-test for normally distributed numerical variables \& Chi-Square statistics for categorical variables. AL: anastomotic leakage; $\mathrm{AH}$ : anastomotic healing; CRCA: colorectal cancer; 
medRxiv preprint doi: https://doi.org/10.1101/2021.10.25.21265372; this version posted October 26, 2021. The copyright holder for this preprint (which was not certified by peer review) is the author/funder, who has granted medRxiv a license to display the preprint in It is made available under a CC-BY-NC-ND 4.0 International license.

M: males; F: females; BMI: body mass index (see also Figure S2); NSAID: non-steroidal anti-inflammatory drugs treatment before surgery; ASA: American society of anesthesiologists; RT: radiotherapy; CRT: chemoradiotherapy; N/A: not applicable; *the remaining patients did not undergo neoadjuvant therapy; ${ }^{2}{ }^{2}(1, N=$ 57) $=0.967, P=0.002$ 
medRxiv preprint doi: https://doi.org/10.1101/2021.10.25.21265372; this version posted October $26,2021$. The copyright holder for this preprint (which was not certified by peer review) is the author/funder, who has granted medRxiv a license to display the preprint in It is made available under a CC-BY-NC-ND 4.0 International license.

Figure 1. Preoperative abundance of Prevotella associates with AH after colorectal surgery in the absence of profound preoperative AL-related fecal microbial dysbiosis.

A

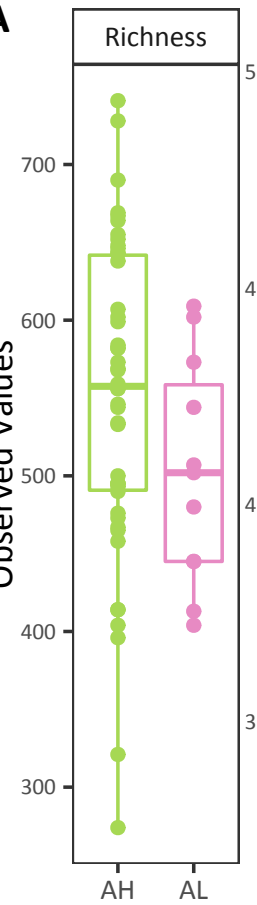

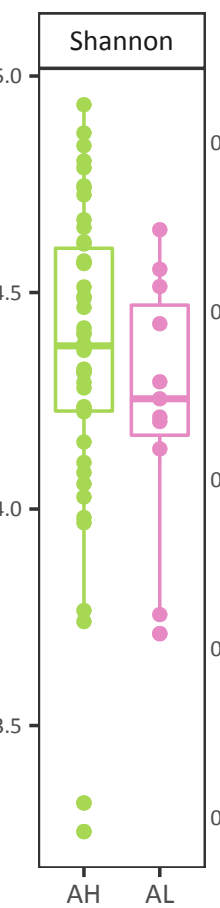
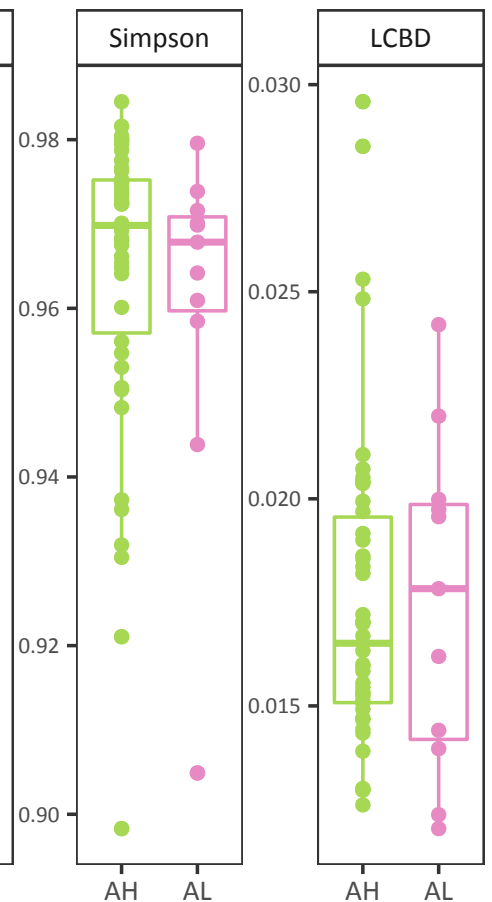

C

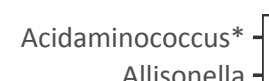
Allisonella Butyrivibrio*CAG-352

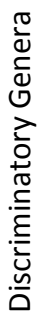
Fusobacterium Lachnospiraceae UCG-001 Megamonas 7 Rikenellaceae RC9 gut group Ruminococcaceae UCG-010unclassified Prevotellaceae
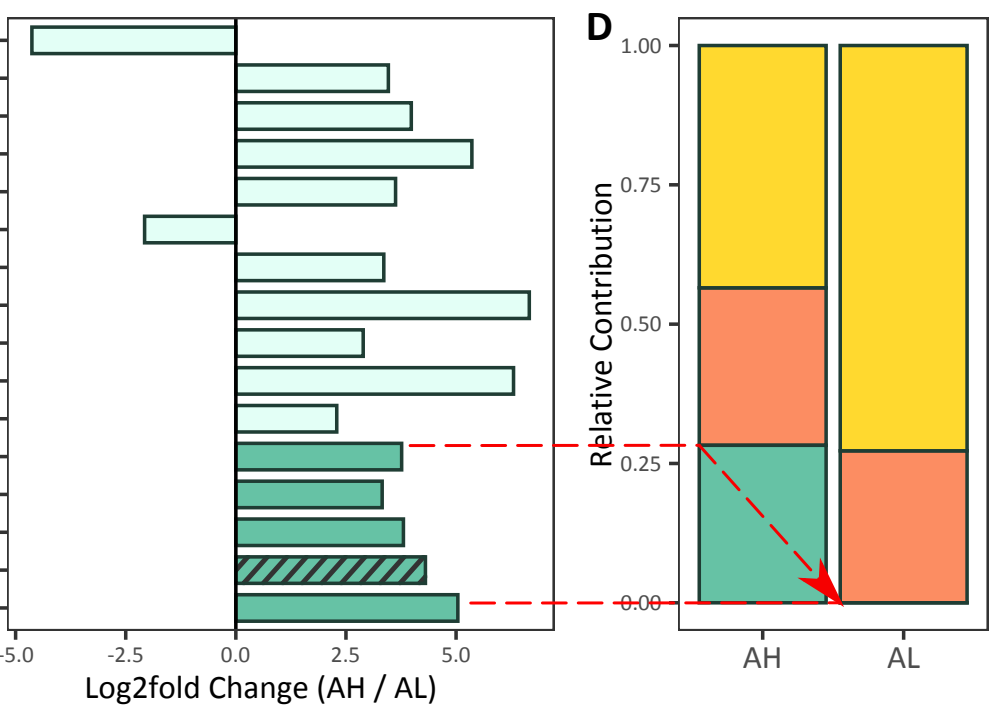

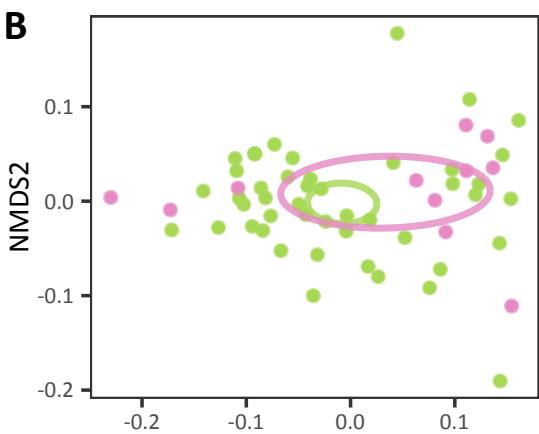

Groups

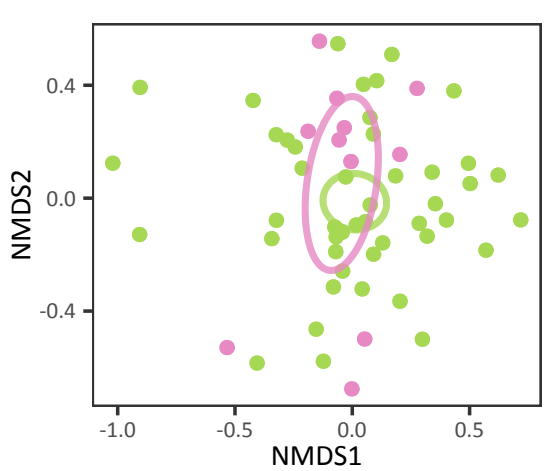

Enterotype

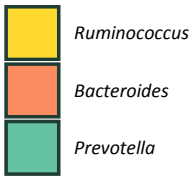


medRxiv preprint doi: https://doi.org/10.1101/2021.10.25.21265372; this version posted October 26, 2021. The copyright holder for this preprint (which was not certified by peer review) is the author/funder, who has granted medRxiv a license to display the preprint in It is made available under a CC-BY-NC-ND 4.0 International license.

Table S1. Bioenv selected taxa explain $\mathbf{8 3 . 5 \%}$ of the variance in fecal microbiota structure of preoperative patients described by the complete ASV dataset agglomerated at genus level.

\section{Faecalibacterium}

Subdoligranulum

Ruminococcus 2

Unclassified Ruminococcaceae

Unclassified Enterobacteriaceae

Akkermansia

Prevotella $9 *$

Bacteroides

Bifidobacterium

Ruminococcaceae UCG-014

Unknown

Unclassified Lachnospiraceae

Methanobrevibacter

Phascolarctobacterium

Acidaminococcus*

Treponema 2

Parabacteroides

Christensenellaceae R-7 group

[Eubacterium] ruminantium group

Butyrivibrio*

Streptococcus

Asterisk indicates taxa with significantly different relative expression between AL and AH groups (DESeq2 analysis presented in Table S2). 
medRxiv preprint doi: https://doi.org/10.1101/2021.10.25.21265372; this version posted October $26,2021$. The copyright holder for this preprint (which was not certified by peer review) is the author/funder, who has granted medRxiv a license to display the preprint in It is made available under a CC-BY-NC-ND 4.0 International license

Table S2. Taxa with significantly different relative abundance in preoperative fecal samples of $A L$ and $A H$ patients; DESeq2 analysis at ASV and genus level.

\begin{tabular}{|c|c|c|c|c|}
\hline & baseMean & $\begin{array}{l}\text { log2-Fold } \\
\text { Change }\end{array}$ & $\boldsymbol{P}$ & $P$ (adjusted) \\
\hline \multicolumn{5}{|l|}{ ASV level } \\
\hline ASV-0557 Acidaminococcus & 24.53 & -5.67 & $3.94 \mathrm{E}-15$ & 4.71E-12 \\
\hline ASV-0190 unclassified Lachnospiraceae & 15.41 & -4.80 & $1.39 \mathrm{E}-14$ & $8.28 \mathrm{E}-12$ \\
\hline ASV-2603 unclassified Lachnospiraceae & 5.86 & -3.87 & $4.36 \mathrm{E}-13$ & $1.74 \mathrm{E}-10$ \\
\hline ASV-1379 Alistipes & 3.91 & -3.65 & $2.98 \mathrm{E}-12$ & $8.90 \mathrm{E}-10$ \\
\hline ASV-0294 Tyzzerella & 86.45 & 6.16 & $7.41 \mathrm{E}-10$ & $1.37 \mathrm{E}-07$ \\
\hline ASV-0333 Ruminococcaceae UCG-014 & 153.59 & 6.37 & $7.99 \mathrm{E}-10$ & $1.37 \mathrm{E}-07$ \\
\hline ASV-2639 unclassified Lachnospiraceae & 13.35 & -4.02 & $7.66 \mathrm{E}-10$ & $1.37 \mathrm{E}-07$ \\
\hline ASV-0852 unclassified Root & 63.13 & 5.91 & $6.28 \mathrm{E}-09$ & $9.39 E-07$ \\
\hline ASV-1667 unclassified Enterobacteriaceae & 6.52 & -3.53 & $2.78 \mathrm{E}-08$ & 3.69E-06 \\
\hline ASV-0219 unclassified Lachnospiraceae & 8.29 & -3.34 & $3.64 \mathrm{E}-08$ & 4.35E-06 \\
\hline ASV-1294 Prevotella 9 & 79.19 & 5.24 & $6.81 \mathrm{E}-08$ & 7.40E-06 \\
\hline ASV-1692 unclassified Enterobacteriaceae & 26.69 & -4.22 & $8.06 \mathrm{E}-08$ & 7.62E-06 \\
\hline ASV-2086 [eubacterium] oxidoreducens group & 50.67 & 4.59 & $8.28 \mathrm{E}-08$ & 7.62E-06 \\
\hline ASV-0875 Bacteroides & 50.51 & 5.29 & $1.64 \mathrm{E}-07$ & $1.40 \mathrm{E}-05$ \\
\hline ASV-1535 Odoribacter & 3.16 & -2.81 & $2.73 \mathrm{E}-07$ & $2.18 \mathrm{E}-05$ \\
\hline ASV-2157 CAG-352 & 34.95 & 4.94 & $4.18 \mathrm{E}-07$ & $3.12 \mathrm{E}-05$ \\
\hline ASV-1594 Rikenellaceae RC9 gut group & 27.33 & 4.94 & $5.04 \mathrm{E}-07$ & $3.55 \mathrm{E}-05$ \\
\hline ASV-1326 Prevotella 9 & 20.72 & 4.53 & $7.75 \mathrm{E}-07$ & 5.15E-05 \\
\hline ASV-0383 Ruminococcaceae UCG-014 & 32.57 & 4.74 & $8.46 \mathrm{E}-07$ & 5.33E-05 \\
\hline ASV-2820 Butyrivibrio & 25.12 & 4.69 & $1.15 \mathrm{E}-06$ & $6.85 \mathrm{E}-05$ \\
\hline ASV-0038 unclassified Lachnospiraceae & 27.70 & 3.76 & $2.87 \mathrm{E}-06$ & $1.64 e-04$ \\
\hline ASV-0691 Holdemanella & 124.12 & 4.19 & $6.57 \mathrm{E}-06$ & $3.27 e-04$ \\
\hline ASV-0937 unclassified Bacteroidales & 15.50 & 3.87 & $6.17 \mathrm{E}-06$ & $3.27 e-04$ \\
\hline ASV-1319 Prevotella 9 & 1471.13 & 4.45 & $6.57 \mathrm{E}-06$ & $3.27 e-04$ \\
\hline ASV-0396 unclassified Clostridiales & 162.44 & 4.06 & $7.35 \mathrm{E}-06$ & $3.52 \mathrm{e}-04$ \\
\hline ASV-1385 Alistipes & 17.60 & 3.28 & $1.25 \mathrm{E}-05$ & $5.76 e-04$ \\
\hline ASV-1344 Prevotella 9 & 21.31 & 3.63 & $1.82 \mathrm{E}-05$ & 7.79e-04 \\
\hline ASV-1266 Prevotella 2 & 21.02 & 3.92 & $2.11 \mathrm{E}-05$ & $8.5 e-04$ \\
\hline ASV-1853 Christensenellaceae R-7 group & 14.83 & 3.41 & $2.13 \mathrm{E}-05$ & $8.5 e-04$ \\
\hline ASV-0756 Bifidobacterium & 46.22 & 3.47 & $2.56 \mathrm{E}-05$ & $9.89 e-04$ \\
\hline ASV-0157 unclassified Lachnospiraceae & 11.03 & 3.37 & $3.02 \mathrm{E}-05$ & $1.13 e-03$ \\
\hline ASV-2097 unclassified Bacteria & 10.10 & 3.48 & $4.48 \mathrm{E}-05$ & $1.58 \mathrm{e}-03$ \\
\hline ASV-2491 unclassified Lachnospiraceae & 5.25 & -2.38 & 4.36E-05 & $1.58 \mathrm{e}-03$ \\
\hline ASV-1290 Prevotella 9 & 14.52 & 3.47 & $6.85 \mathrm{E}-05$ & $2.34 \mathrm{e}-03$ \\
\hline ASV-1460 Parabacteroides & 3.87 & -2.11 & 7.29E-05 & $2.42 e-03$ \\
\hline ASV-0078 Lachnospiraceae UCG-004 & 28.35 & 2.96 & $7.52 \mathrm{E}-05$ & $2.43 e-03$ \\
\hline ASV-0888 unclassified Bacteroidales & 23.31 & 3.63 & $9.23 \mathrm{E}-05$ & $2.83 e-03$ \\
\hline ASV-2025 Ruminiclostridium 9 & 32.08 & 2.86 & $1.10 \mathrm{e}-04$ & $3.28 \mathrm{e}-03$ \\
\hline
\end{tabular}


medRxiv preprint doi: https://doi.org/10.1101/2021.10.25.21265372; this version posted October 26, 2021. The copyright holder for this preprint (which was not certified by peer review) is the author/funder, who has granted medRxiv a license to display the preprint in It is made available under a CC-BY-NC-ND 4.0 International license

\begin{tabular}{|c|c|c|c|c|}
\hline \multicolumn{5}{|l|}{ Continued } \\
\hline ASV-0014 unclassified Lachnospiraceae & 10.04 & -2.32 & $1.42 \mathrm{e}-04$ & $3.78 \mathrm{e}-03$ \\
\hline ASV-0441 unclassified Clostridiales & 16.22 & 3.22 & $1.42 \mathrm{e}-04$ & $3.78 \mathrm{e}-03$ \\
\hline ASV-1098 Bacteroides & 74.99 & 2.88 & $1.41 \mathrm{e}-04$ & $3.78 \mathrm{e}-03$ \\
\hline ASV-1800 Desulfovibrio & 9.48 & 3.39 & $1.33 e-04$ & $3.78 \mathrm{e}-03$ \\
\hline ASV-1623 Akkermansia & 10.69 & 3.32 & $1.64 \mathrm{e}-4$ & $4.23 e-03$ \\
\hline ASV-2823 Butyrivibrio & 9.42 & 3.38 & $1.66 \mathrm{e}-04$ & $4.23 e-03$ \\
\hline ASV-1340 unclassified Bacteroidales & 18.90 & 3.09 & $1.91 \mathrm{e}-04$ & $4.66 \mathrm{e}-03$ \\
\hline ASV-2815 Lachnoclostridium & 11.88 & 2.76 & $1.99 e-04$ & $4.76 \mathrm{e}-03$ \\
\hline ASV-1619 unclassified Root & 8.81 & 3.03 & $2.04 \mathrm{e} 04$ & $4.78 \mathrm{e}-03$ \\
\hline ASV-0562 Megamonas & 8.37 & 3.20 & $2.85 e-04$ & $6.31 \mathrm{e}-03$ \\
\hline ASV-0326 Ruminococcaceae UCG-014 & 6.51 & 2.83 & $2.91 e-04$ & $6.34 \mathrm{e}-03$ \\
\hline ASV-2011 Ruminococcaceae UCG-003 & 8.99 & 2.42 & $3.21 \mathrm{e}-04$ & $6.86 e-03$ \\
\hline ASV-0164 Lachnospiraceae UCG-001 & 103.64 & -2.82 & $4.80 e-04$ & $9.25 e-03$ \\
\hline ASV-0426 unclassified Root & 6.26 & 2.64 & $4.67 e-04$ & $9.25 e-03$ \\
\hline ASV-1045 Bacteroides & 7.98 & 3.00 & $4.56 e-04$ & $9.25 e-03$ \\
\hline ASV-1156 Bacteroides & 11.00 & 2.52 & $4.53 e-04$ & $9.25 e-03$ \\
\hline ASV-2564 [eubacterium] eligens group & 23.77 & 2.33 & $4.64 e-04$ & $9.25 e-03$ \\
\hline ASV-0425 unclassified Root & 11.34 & 2.93 & $4.98 e-04$ & $9.45 e-03$ \\
\hline ASV-0376 Ruminococcaceae UCG-014 & 7.12 & 2.96 & $5.45 \mathrm{E}-04$ & $1.00 \mathrm{e}-02$ \\
\hline ASV-0241 unclassified Lachnospiraceae & 10.43 & -2.30 & $5.82 e-04$ & $1.05 e-02$ \\
\hline ASV-2445 Ruminiclostridium 6 & 23.35 & 2.85 & $5.87 e-04$ & $1.05 e-02$ \\
\hline ASV-1213 Paraprevotella & 71.60 & 3.03 & $6.16 e-04$ & $1.08 \mathrm{e}-02$ \\
\hline ASV-1312 Prevotella 9 & 8.22 & 2.62 & $6.68 \mathrm{e}-04$ & $1.16 \mathrm{e}-02$ \\
\hline ASV-2103 unclassified Clostridiales & 5.88 & 2.68 & $7.26 \mathrm{e}-04$ & $1.24 \mathrm{e}-02$ \\
\hline ASV-1300 Prevotella 9 & 9.46 & 2.57 & $7.41 \mathrm{e} 04$ & $1.25 \mathrm{e}-02$ \\
\hline ASV-2509 [eubacterium] xylanophilum group & 5.28 & 2.52 & 7.91e-04 & $1.31 \mathrm{e}-02$ \\
\hline ASV-0315 Ruminococcaceae UCG-014 & 5.84 & 2.67 & $9.16 e-04$ & $1.46 \mathrm{e}-02$ \\
\hline ASV-1964 Ruminococcaceae UCG-002 & 17.29 & 2.61 & $9.12 \mathrm{e}-04$ & $1.46 \mathrm{e}-02$ \\
\hline ASV-0269 unclassified Lachnospiraceae & 6.44 & 2.45 & $1.04 \mathrm{e}-03$ & $1.64 \mathrm{e}-02$ \\
\hline ASV-0395 unclassified Clostridiales & 5.17 & 2.49 & $1.15 e-03$ & $1.77 e-02$ \\
\hline ASV-2459 unclassified Ruminococcaceae & 5.85 & 2.54 & $1.17 e-03$ & $1.77 \mathrm{e}-02$ \\
\hline ASV-0357 Ruminococcaceae UCG-014 & 69.78 & 3.09 & $1.21 \mathrm{e}-03$ & $1.82 \mathrm{e}-02$ \\
\hline ASV-0233 unclassified Lachnospiraceae & 93.30 & -2.16 & $1.26 \mathrm{e}-03$ & $1.85 e-02$ \\
\hline ASV-0866 Bacteroides & 6.30 & 2.78 & $1.27 e-03$ & $1.85 e-02$ \\
\hline ASV-2118 Ruminococcaceae UCG-010 & 4.75 & 2.36 & $1.28 \mathrm{e}-03$ & $1.85 e-02$ \\
\hline ASV-2109 Ruminococcaceae UCG-010 & 5.66 & 2.62 & $1.40 \mathrm{e}-03$ & $2.00 \mathrm{e}-02$ \\
\hline ASV-1330 Prevotella 9 & 4.78 & 2.37 & $1.43 e-03$ & $2.01 \mathrm{e}-02$ \\
\hline ASV-1315 Prevotella 9 & 22.38 & 2.68 & $1.49 e-03$ & 2.07e-02 \\
\hline ASV-0808 Libanicoccus & 4.70 & 2.35 & $1.53 e-03$ & $2.11 \mathrm{e}-02$ \\
\hline ASV-1311 Prevotella 9 & 23.29 & 2.63 & $1.64 \mathrm{e}-03$ & $2.21 \mathrm{e}-02$ \\
\hline ASV-1890 Ruminococcaceae UCG-013 & 13.03 & 2.52 & $1.63 e-03$ & $2.21 \mathrm{e}-02$ \\
\hline ASV-1353 Alistipes & 55.51 & 2.85 & $1.68 e-03$ & $2.24 \mathrm{e}-02$ \\
\hline
\end{tabular}


medRxiv preprint doi: https://doi.org/10.1101/2021.10.25.21265372; this version posted October $26,2021$. The copyright holder for this preprint (which was not certified by peer review) is the author/funder, who has granted medRxiv a license to display the preprint in It is made available under a CC-BY-NC-ND 4.0 International license

\begin{tabular}{|c|c|c|c|c|}
\hline \multicolumn{5}{|l|}{ Continued } \\
\hline ASV-1085 Bacteroides & 33.38 & 2.43 & $1.74 \mathrm{e}-03$ & $2.29 \mathrm{e}-02$ \\
\hline ASV-1322 Prevotella 9 & 5.08 & 2.33 & $1.79 e-03$ & $2.33 e-02$ \\
\hline ASV-0892 Bacteroides & 5.55 & 2.59 & $1.84 \mathrm{e}-03$ & $2.37 e-02$ \\
\hline ASV-0630 Streptococcus & 44.73 & -2.18 & $1.91 \mathrm{e}-03$ & $2.43 e-02$ \\
\hline ASV-2211 unclassified Ruminococcaceae & 7.02 & 2.13 & $1.96 \mathrm{e}-03$ & $2.44 e-02$ \\
\hline ASV-1118 Bacteroides & 4.25 & 2.19 & $2.00 \mathrm{e}-03$ & $2.46 \mathrm{e}-02$ \\
\hline ASV-0370 Ruminococcaceae UCG-014 & 21.86 & 2.61 & $2.05 e-03$ & $2.48 \mathrm{e}-02$ \\
\hline ASV-1772 Sutterella & 5.43 & 2.43 & $2.05 e-03$ & $2.48 \mathrm{e}-02$ \\
\hline ASV-1420 Parabacteroides & 28.49 & 2.16 & $2.11 \mathrm{e}-03$ & $2.53 \mathrm{e} 02$ \\
\hline ASV-1555 Barnesiella & 4.65 & 2.19 & 2.17e-03 & $2.56 \mathrm{e}-02$ \\
\hline ASV-1599 unclassified Rikenellaceae & 4.70 & 2.49 & $2.24 \mathrm{e}-03$ & $2.60 \mathrm{e}-02$ \\
\hline ASV-1602 Rikenellaceae RC9 gut group & 5.58 & 2.60 & $2.32 \mathrm{e}-03$ & $2.67 e-02$ \\
\hline ASV-0286 Tyzzerella 3 & 4.47 & 2.27 & $2.44 \mathrm{e}-03$ & $2.75 e-02$ \\
\hline ASV-1865 Christensenellaceae R-7 group & 11.23 & 2.26 & $2.49 \mathrm{e}-03$ & $2.78 \mathrm{e}-02$ \\
\hline ASV-0530 Phascolarctobacterium & 5.15 & 2.12 & $2.72 \mathrm{e}-03$ & 2.99e-02 \\
\hline ASV-0624 Streptococcus & 5.19 & 2.49 & $2.72 \mathrm{e}-03$ & 2.99e-02 \\
\hline ASV-1165 Bacteroides & 5.39 & 2.08 & $2.88 \mathrm{e}-03$ & $3.11 \mathrm{e}-02$ \\
\hline ASV-1843 Christensenellaceae R-7 group & 88.23 & 2.20 & $2.91 \mathrm{e}-03$ & $3.11 \mathrm{e}-02$ \\
\hline ASV-2164 Ruminococcus 2 & 16.41 & 2.32 & $3.28 \mathrm{e}-03$ & 3.47e-02 \\
\hline ASV-1350 Prevotella 9 & 4.97 & 2.30 & $3.46 \mathrm{e}-03$ & $3.60 \mathrm{e}-02$ \\
\hline ASV-2177 unclassified Ruminococcaceae & 5.15 & 2.01 & $3.43 e-03$ & $3.60 \mathrm{e}-02$ \\
\hline ASV-0453 Family XIII AD3011 group & 10.48 & 2.14 & $3.54 \mathrm{e}-03$ & $3.64 \mathrm{e}-02$ \\
\hline ASV-0183 unclassified Lachnospiraceae & 15.50 & 2.05 & $3.93 e-03$ & 3.99e-02 \\
\hline ASV-0494 unclassified Clostridiaceae 1 & 18.10 & 2.36 & $3.97 e-03$ & $3.99 \mathrm{e}-02$ \\
\hline ASV-2171 Ruminococcus 2 & 3.80 & 2.02 & $4.87 e-03$ & $4.78 \mathrm{e}-02$ \\
\hline ASV-0346 Ruminococcaceae UCG-014 & 4.55 & 2.29 & $5.01 \mathrm{e}-03$ & $4.78 \mathrm{e}-02$ \\
\hline \multicolumn{5}{|l|}{ Genus level } \\
\hline Tyzzerella & 108.36 & 6.30 & $4.23 \mathrm{E}-10$ & 3.93E-08 \\
\hline Rikenellaceae RC9 gut group* & 126.26 & 6.67 & $2.33 \mathrm{E}-10$ & $3.93 \mathrm{E}-08$ \\
\hline Acidaminococcus & 34.38 & -4.63 & $7.50 \mathrm{E}-09$ & $4.65 \mathrm{E}-07$ \\
\hline unclassified Prevotellaceae & 75.95 & 5.05 & $3.42 \mathrm{E}-08$ & $1.59 \mathrm{E}-06$ \\
\hline CAG-352 & 53.14 & 5.36 & 7.17E-08 & 2.67E-06 \\
\hline Allisonella & 11.30 & 3.46 & $2.20 \mathrm{E}-05$ & 5.59E-04 \\
\hline Prevotella 9 & 1977.76 & 4.32 & $2.40 \mathrm{E}-05$ & 5.59E-04 \\
\hline Butyrivibrio & 45.82 & 4.01 & $2.11 \mathrm{E}-05$ & $5.59 \mathrm{E}-04$ \\
\hline Prevotella 2 & 26.29 & 3.83 & $3.59 \mathrm{E}-05$ & $7.42 \mathrm{E}-04$ \\
\hline Fusobacterium & 36.74 & 3.63 & $6.47 \mathrm{E}-05$ & $1.20 \mathrm{E}-03$ \\
\hline Ruminococcaceae UCG-010* & 110.18 & 2.90 & $7.35 \mathrm{E}-05$ & $1.24 \mathrm{E}-03$ \\
\hline Megamonas & 9.57 & 3.36 & 2.09E-04 & $3.15 \mathrm{E}-03$ \\
\hline Paraprevotella & 112.14 & 3.32 & $2.20 \mathrm{E}-04$ & $3.15 \mathrm{E}-03$ \\
\hline Alloprevotella & 147.70 & 3.76 & $3.03 \mathrm{E}-04$ & $4.03 E-03$ \\
\hline Lachnospiraceae UCG-001 & 170.75 & -2.07 & 2.07E-03 & $2.26 \mathrm{E}-02$ \\
\hline
\end{tabular}


medRxiv preprint doi: https://doi.org/10.1101/2021.10.25.21265372; this version posted October 26, 2021. The copyright holder for this preprint (which was not certified by peer review) is the author/funder, who has granted medRxiv a license to display the preprint in It is made available under a CC-BY-NC-ND 4.0 International license

\section{Continued}

Tyzzerella 3

5.16

2.29

2.69E-03

2.64E-02

Log2-fold change is negative when the relative abundance of ASVs belonging to the respective genus is higher in the AL group. 
medRxiv preprint doi: https://doi.org/10.1101/2021.10.25.21265372; this version posted October 26, 2021. The copyright holder for this preprint (which was not certified by peer review) is the author/funder, who has granted medRxiv a license to display the preprint in It is made available under a CC-BY-NC-ND 4.0 International license.

\section{Figure S1. No difference found in the colonic tissue transcriptional profile of patients who undergo colorectal}

surgery and develop AL. (A) Volcano plot of genes in AL compared to AH patients indicates the absence of significant DEG at surgery. (B) Heatmap representation of the hierarchical clustering analysis of the 150 most variable gene suggests no clear clustering between $\mathrm{AL}$ and $\mathrm{AH}$ patients.

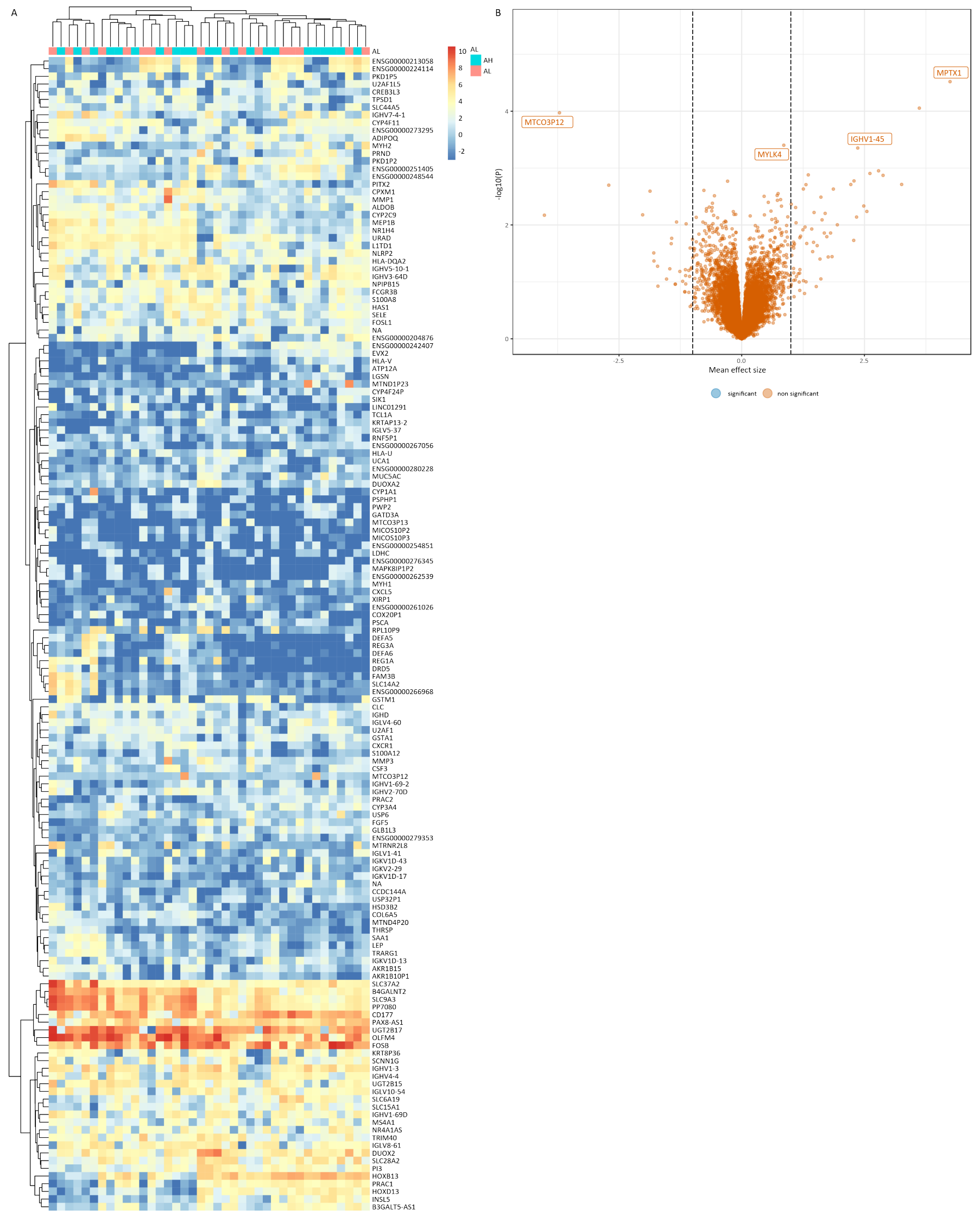


medRxiv preprint doi: https://doi.org/10.1101/2021.10.25.21265372; this version posted October 26, 2021. The copyright holder for this preprint (which was not certified by peer review) is the author/funder, who has granted medRxiv a license to display the preprint in It is made available under a CC-BY-NC-ND 4.0 International license.

Figure S2. Lack of significant correlation between the microbial discriminatory and preoperative individual components suggests their AL-specific nature. Kendall rank correlations between the relative abundance of the 16 discriminative genera, participants characteristics and preoperative serum cytokines concentrations; Benjamini-Hochberg correction was applied for multiple testing.

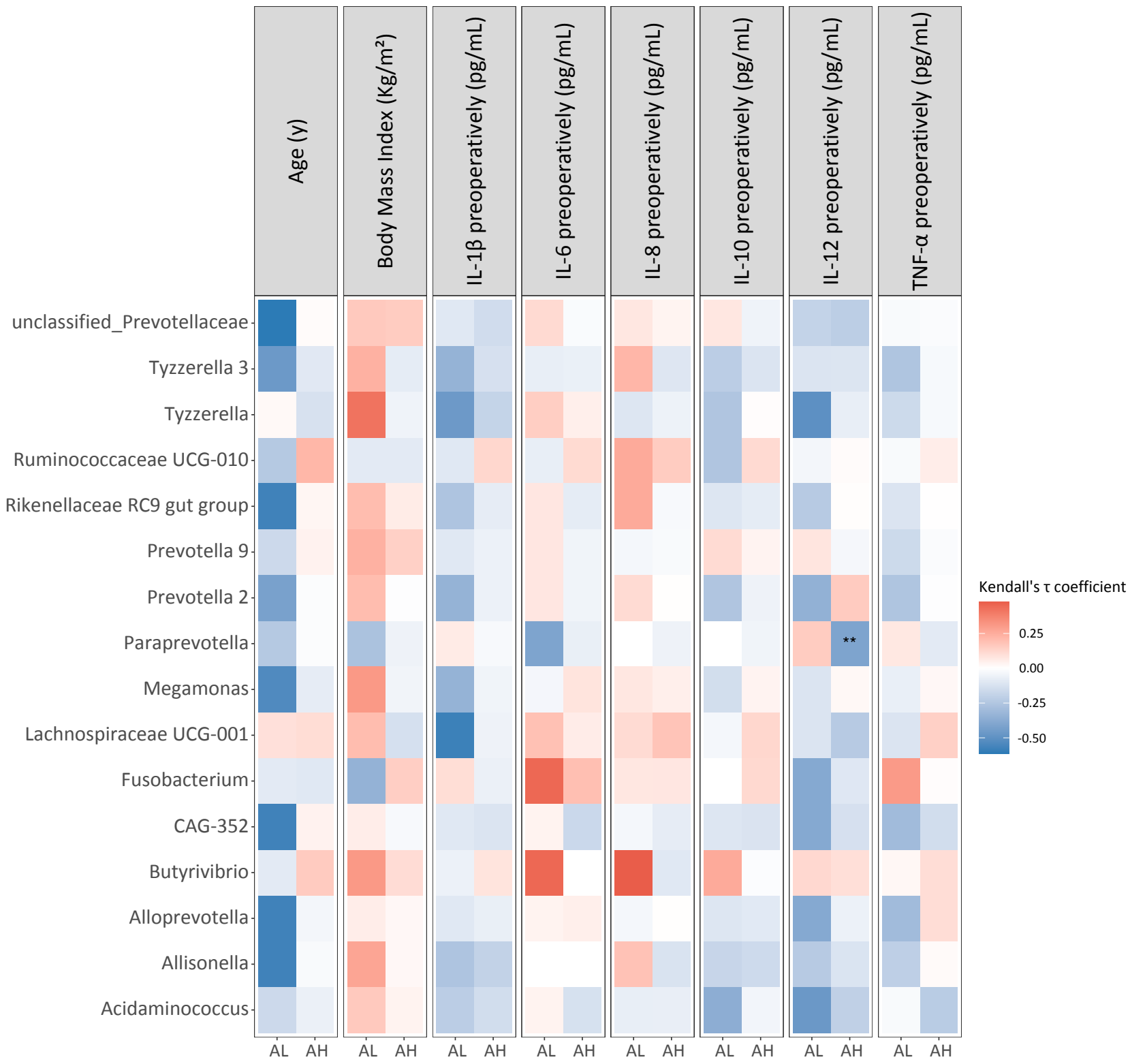


medRxiv preprint doi: https://doi.org/10.1101/2021.10.25.21265372; this version posted October 26, 2021. The copyright holder for this preprint (which was not certified by peer review) is the author/funder, who has granted medRxiv a license to display the preprint in It is made available under a CC-BY-NC-ND 4.0 International license .

\section{Supplemental methods}

\section{Human subjects}

All subjects included in the present study were recruited in the SANICSII trial, which was open for inclusion from August 2014 until February 2017, performed to compare perioperative nutrition to standard care with POI as primary endpoint. ASA physical status score was used to assess their fitness before surgery. C-reactive protein was measured to evaluate inflammation postoperatively with four consecutive daily measurements. AL was defined radiologically or confirmed during re-operation. Patients who developed $A L$ were treated with antibiotics, drainage, secondary surgery or untreated in the absence of symptoms.

In total 280 patients were included, all of which had provided a written informed consent. The current study pertained a subset of 36 patients used to interrogate the anastomotic-tissue associated transcriptome at the time of surgery, and a subset of 57 patients used to evaluate the preoperative fecal microbiota. Information about all patients' age, sex, BMI and postoperative complications (AL or AH) is provided in Table 1. Further detailed information about patients' pre- (i.e. NSAID treatment, ASA class and neoadjuvant therapy), postoperative (i.e., AL diagnosis and treatment) metadata, and surgery's characteristics (i.e., colonic or rectal resection, laparoscopic, open surgery or conversion to open surgery, and formation of colostomy during surgery) are also reported in Table 1; as no significant difference in the host transcriptome between $\mathrm{AL}$ and $\mathrm{AH}$ patients, detailed information about pre-, peri- and postoperative metadata are only reported for the 57 patients included in the fecal microbiota cohort.

\section{RNA sequencing}

RNA was isolated from human full thickness colonic tissue using the Isolate II RNA mini kit (Cat\# BIO-52073, Meridian Bioscience). RNA quality was assured using the Bioanalyzer (Agilent, Santa Clara, USA), including only samples with a RIN score higher than 8. Conversion of the mRNA to cDNA was done using the NEBNext Ultra Directional RNA Library Prep Kit (Cat\# E7760L, New England Biolabs) for Illumina and subsequent sequencing of the cDNA was performed on the NextSeq500 to a depth of $10 \mathrm{M}$ reads per sample. Both cDNA conversion and sequencing were performed at GenomeScan (Leiden, The Netherlands).

Quality control of the raw reads was done with FastQC (version 0.11.5) (Andrews, 2010) and the summarization thereof through MultiQC (version 0.8) (Ewels et al., 2016). Raw reads were aligned to the human genome (GRCh38) using STAR (version 2.5.3a) (Dobin et al., 2013) and annotated using the Ensembl v91 annotation (Aken et al., 2017). Post-alignment processing was performed through SAMtools (version 1.2) (Li et al., 2009), after which reads were counted using the featureCounts application in the Subread package (version 1.6.0) (Liao et al., 2013).

\section{Fecal sample collection}

Feces were collected by the patients on the day prior to surgery and immediately stored at $-20^{\circ} \mathrm{C}$.

Microbiota profiling 
medRxiv preprint doi: https://doi.org/10.1101/2021.10.25.21265372; this version posted October $26,2021$. The copyright holder for this preprint (which was not certified by peer review) is the author/funder, who has granted medRxiv a license to display the preprint in It is made available under a CC-BY-NC-ND 4.0 International license .

DNA was extracted from $150 \mathrm{mg}$ of fecal sample using mechanical lysis and the PSP Spin Stool DNA Plus Kit (Cat\# 1038110300, STRATEC Molecular GmbH) isolation method. The samples were placed in Lysing Matrix E tubes with 1,400 ul of stool stabilizer from the PSP kit. Mechanical lysis was done using the FastPrep at 3 repetitive rounds of $30 \mathrm{sec}$ at $6.5 \mathrm{~m} / \mathrm{s}$, with cooling of $30 \mathrm{sec}$ on ice in between. After the FastPrep procedure further extraction was done at $95^{\circ} \mathrm{C}$ for $15 \mathrm{~min}$ followed by cooling on ice for $1 \mathrm{~min}$ and centrifugation at $13,400 \mathrm{~g}$ for $1 \mathrm{~min}$. The supernatant was transferred to the PSP InviAdsorb. Negative extraction controls (DNA-free water) were processed in the same way. The DNA concentration was measured with the Nanodrop 1000 spectrophotometer (Thermo Fisher Scientific).

$16 \mathrm{~S}$ rRNA gene amplicons were generated using a single step PCR protocol targeting the V3-V4 region (Kozich et al., 2013); both forward and reverse primers are listed in the key resources table. The PCR reaction in $20 \mathrm{ul}$ was performed with following thermocycling conditions: initial denaturation at $98^{\circ} \mathrm{C}$ followed by 25 cycles denaturation $\left(10 \mathrm{sec}\right.$ at $\left.98^{\circ} \mathrm{C}\right)$, annealing $\left(20 \mathrm{sec}\right.$ at $\left.55^{\circ} \mathrm{C}\right)$ and extension $\left(90 \mathrm{sec}\right.$ at $\left.72^{\circ} \mathrm{C}\right)$ and final extension at $72^{\circ} \mathrm{C}$ for $10 \mathrm{~min}$. PCR products were purified using Ampure XP beads (Cat\# A63882, Beckman Coulter Life Sciences) and purified products were equimolar pooled. The libraries were sequenced using a MiSeq platform using V3 chemistry with 2×251 cycles.

Forward and reverse reads were truncated to 240 and 210 bases respectively and merged using USEARCH; merged reads that did not pass the Illumina chastity filter, had an expected error rate higher than 2, or were shorter than 380 bases were filtered. Amplified sequence variants (ASVs) were inferred for each sample individually with a minimum abundance of 4 reads (Edgar, 2016). Unfiltered reads were then mapped against the collective ASV set to determine the abundances. Taxonomy was assigned using SILVA $16 S$ ribosomal database V132 (Quast et al., 2013). Enterotype classification was done based on the JensenShannon divergence-clustering of the samples, as published by the MetaHIT consortium in 2011 https://enterotype.embl.de/enterotypes.html.

\section{Serum cytokines measurement}

Blood samples were collected from all patients the day before surgery. They were immediately put on ice, centrifuged at $4^{\circ} \mathrm{C}$ and $3,000 \mathrm{~g}$ for $12 \mathrm{~min}$ and the plasma was stored at $-80^{\circ} \mathrm{C}$ until further analysis. To determine the most important inflammatory cytokines, a Human Inflammatory Cytokines Kit was used to measure cytokine levels of IL-13, IL-6, IL-8, IL-10, IL-12, and TNF- $\alpha$ by cytometric bead array (Cat\# 551811, BD Biosciences). Standards of 9 distinct concentrations were used (i.e., 5,000, 25,000, 1,250, 625, 312.5, $156,80,40$ and $20 \mathrm{pg} / \mathrm{mL}$ ), along with a blank sample. Mix bead solution was prepared and incubated with PE detection reagent and the samples for $3 \mathrm{hrs}$, at room temperature, in 96-well plate $\mathrm{V}$ bottom wells. Samples were measured on a BD LSRFortessa (BD, Biosciences) and analyzed using BD Flowjo Software (version 10.7.1).

\section{Quantification and statistical analysis}

Differential expression analysis of the RNA sequencing data was performed using edgeR (version 3.20.6) (Robinson et al., 2010) in the R statistical environment (version 3.4.3). A quasi-likelihood F-test (QL F-test) 
medRxiv preprint doi: https://doi.org/10.1101/2021.10.25.21265372; this version posted October 26, 2021. The copyright holder for this preprint (which was not certified by peer review) is the author/funder, who has granted medRxiv a license to display the preprint in It is made available under a CC-BY-NC-ND 4.0 International license .

was performed using the gImQLFit function to find differentially expressed genes using the estimated trended dispersions; results presented in Figure S1. Regressions were corrected for age and sex.

Clinical metadata were analyzed using IBM SPSS Statistics for Windows (version 26.0); both onesample Kolmogorov-Smirnov and Shapiro-Wilk tests were used to confirm normal distribution in numerical variables. Chi-Square test of Independence was applied to explore associations between categorical variables. Parametric independent-samples t-test was conducted to compare the means of normally distributed variables between the groups. All statistical parameters are reported in Table 1; in the categorical variables, $\mathrm{n}$ represents the exact number of patients and percentage (\%) refers to the respective AL or AH grouping. In the numerical variables, values present the mean, and standard error of the mean follows within brackets.

Microbial multivariate statistical analysis was performed in R statistical environment (version 4.3.0) using the packages phyloseq (version 1.34.0) (McMurdie and Holmes, 2013) (Oksanen J., 2020) and vegan (version 2.5.7). Samples were rarefied to 30,000 reads. Alpha diversity indices were calculated (i.e., species richness, Shannon entropy, Simpson diversity index). Microbial composition was assessed using non-metric multidimensional scaling plots (NMDS) at ASV level based on Bray-Curtis dissimilarity index and unweighted UniFrac distance. The former considers bacterial taxon abundance, while the latter considers phylogenetic distance between bacterial taxa through presence/absence, regardless of proportional representation. Nonparametric permutational multivariate analysis of variance (PERMANOVA) was applied using the vegan Adonis function on distance matrices (i.e., Bray-Curtis dissimilarity index and unweighted UniFrac distance). Local contribution to $\beta$ diversity (LCBD) analysis was performed to measure the contribution of each sample to the total ASV $\beta$ diversity; samples with high LCBD represent samples that are markedly different from the average $\beta$ diversity of all study samples. Alpha and beta diversity results are presented in Figure $1 \mathrm{~A}$ and $1 \mathrm{~B}$. Differences in ASV and genus relative abundances between groups were found using the DESeq2 method (version 1.30.1) (Love et al., 2014). Basemean, log2-fold change, non-adjusted and adjusted p-values of the aforementioned analyses at ASV and genus levels are reported in Table S2. For correlations Kendall rank correlation was used; results presented in Figure S2. Benjamini-Hochberg correction was applied to cases of multiple testing. Analysis using the Bioenv function in vegan produced subsets of taxa whose Euclidean distance matrices correlate maximally with the Bray-Curtis dissimilarity matrices derived from complete count tables, thus indicating major determinants of community structure; results presented in Table S1. Conventional random forest analysis used the complete count table aggregated at genus level. Models were generated using Log-proportional abundances via the cforest function of the party R package (version 1.3.7), which creates random forests from unbiased classification trees based on a conditional inference framework (Hothorn et al., 2006). Feature importances were evaluated calculating the conditional variance importance; respective results highlighted on Figure $1 \mathrm{C}$. 
medRxiv preprint doi: https://doi.org/10.1101/2021.10.25.21265372; this version posted October 26, 2021. The copyright holder for this preprint (which was not certified by peer review) is the author/funder, who has granted medRxiv a license to display the preprint in It is made available under a CC-BY-NC-ND 4.0 International license .

\section{References}

Aken, B.L., Achuthan, P., Akanni, W., Amode, M.R., Bernsdorff, F., Bhai, J., Billis, K., Carvalho-Silva, D., Cummins, C., Clapham, P., et al. (2017). Ensembl 2017. Nucleic Acids Res 45, D635-d642. 10.1093/nar/gkw1104. Andrews, S. (2010). FastQC: a quality control tool for highthroughput sequence data.

Arumugam, M., Raes, J., Pelletier, E., Le Paslier, D., Yamada, T., Mende, D.R., Fernandes, G.R., Tap, J., Bruls, T., Batto, J.-M., et al. (2011). Enterotypes of the human gut microbiome. Nature 473, 174-180. 10.1038/nature09944.

Bosmans, J.W., Jongen, A.C., Bouvy, N.D., and Derikx, J.P. (2015). Colorectal anastomotic healing: why the biological processes that lead to anastomotic leakage should be revealed prior to conducting intervention studies. BMC Gastroenterol 15, 180. 10.1186/s12876-015-0410-3.

Dobin, A., Davis, C.A., Schlesinger, F., Drenkow, J., Zaleski, C., Jha, S., Batut, P., Chaisson, M., and Gingeras, T.R. (2013). STAR: ultrafast universal RNA-seq aligner. Bioinformatics 29, 15-21. 10.1093/bioinformatics/bts635.

Edgar, R.C. (2016). UNOISE2: improved error-correction for Illumina 165 and ITS amplicon sequencing. bioRxiv, 081257. 10.1101/081257.

Ewels, P., Magnusson, M., Lundin, S., and Käller, M. (2016). MultiQC: summarize analysis results for multiple tools and samples in a single report. Bioinformatics 32, 3047-3048. 10.1093/bioinformatics/btw354.

Hammond, J., Lim, S., Wan, Y., Gao, X., and Patkar, A. (2014). The burden of gastrointestinal anastomotic leaks: an evaluation of clinical and economic outcomes. J Gastrointest Surg 18, 1176-1185. 10.1007/s11605014-2506-4.

Hothorn, T., Hornik, K., and Zeileis, A. (2006). Unbiased Recursive Partitioning: A Conditional Inference Framework. Journal of Computational and Graphical Statistics 15, 651-674. 10.1198/106186006X133933.

Jongen, A.C., Bosmans, J.W., Kartal, S., Lubbers, T., Sosef, M., Slooter, G.D., Stoot, J.H., van Schooten, F.J., Bouvy, N.D., and Derikx, J.P. (2016). Predictive Factors for Anastomotic Leakage After Colorectal Surgery: Study Protocol for a Prospective Observational Study (REVEAL Study). JMIR Res Protoc 5, e90. 10.2196/resprot. 5477.

Kozich, J.J., Westcott, S.L., Baxter, N.T., Highlander, S.K., and Schloss, P.D. (2013). Development of a dual-index sequencing strategy and curation pipeline for analyzing amplicon sequence data on the MiSeq Illumina sequencing platform. Appl Environ Microbiol 79, 5112-5120. 10.1128/aem.01043-13.

Lee, J.A., Chico, T.J.A., and Renshaw, S.A. (2018). The triune of intestinal microbiome, genetics and inflammatory status and its impact on the healing of lower gastrointestinal anastomoses. Febs j 285, 1212-1225. 10.1111/febs.14346.

Li, H., Handsaker, B., Wysoker, A., Fennell, T., Ruan, J., Homer, N., Marth, G., Abecasis, G., and Durbin, R. (2009). The Sequence Alignment/Map format and SAMtools. Bioinformatics 25, 2078-2079. 10.1093/bioinformatics/btp352.

Liao, Y., Smyth, G.K., and Shi, W. (2013). The Subread aligner: fast, accurate and scalable read mapping by seed-and-vote. Nucleic Acids Res 41, e108. 10.1093/nar/gkt214. 
medRxiv preprint doi: https://doi.org/10.1101/2021.10.25.21265372; this version posted October 26, 2021. The copyright holder for this preprint (which was not certified by peer review) is the author/funder, who has granted medRxiv a license to display the preprint in It is made available under a CC-BY-NC-ND 4.0 International license .

Love, M.I., Huber, W., and Anders, S. (2014). Moderated estimation of fold change and dispersion for RNAseq data with DESeq2. Genome Biology 15, 550. 10.1186/s13059-014-0550-8.

McMurdie, P.J., and Holmes, S. (2013). phyloseq: An R Package for Reproducible Interactive Analysis and Graphics of Microbiome Census Data. PLOS ONE 8, e61217. 10.1371/journal.pone.0061217.

Oksanen J., B.F.G., Friendly M., Kindt R., Legendre P., McGlinn D., Minchin P.R., O'Hara R. B. , Simpson G.L., Solymos P, Stevens M.H.H., Szoecs E. and Wagner H. (2020). vegan: Community Ecology Package. R package version 2.5-7.

Peters, E.G., Dekkers, M., van Leeuwen-Hilbers, F.W., Daams, F., Hulsewé, K.W.E., de Jonge, W.J., Buurman, W.A., and Luyer, M.D.P. (2017). Relation between postoperative ileus and anastomotic leakage after colorectal resection: a post hoc analysis of a prospective randomized controlled trial. Colorectal Dis 19, 667-674. 10.1111/codi.13582.

Peters, E.G., Smeets, B.J.J., Nors, J., Back, C.M., Funder, J.A., Sommer, T., Laurberg, S., Løve, U.S., Leclercq, W.K.G., Slooter, G.D., et al. (2018). Perioperative lipid-enriched enteral nutrition versus standard care in patients undergoing elective colorectal surgery (SANICS II): a multicentre, double-blind, randomised controlled trial. Lancet Gastroenterol Hepatol 3, 242-251. 10.1016/s2468-1253(18)30031-1.

Quast, C., Pruesse, E., Yilmaz, P., Gerken, J., Schweer, T., Yarza, P., Peplies, J., and Glöckner, F.O. (2013). The SILVA ribosomal RNA gene database project: improved data processing and web-based tools. Nucleic Acids Res 41, D590-596. 10.1093/nar/gks1219.

Robinson, M.D., McCarthy, D.J., and Smyth, G.K. (2010). edgeR: a Bioconductor package for differential expression analysis of digital gene expression data. Bioinformatics 26, 139-140. 10.1093/bioinformatics/btp616.

Sahami, S., Buskens, C.J., Fadok, T.Y., Tanis, P.J., de Buck van Overstraeten, A., Wolthuis, A.M., Bemelman, W.A., and D'Hoore, A. (2016). Defunctioning lleostomy is not Associated with Reduced Leakage in Proctocolectomy and Ileal Pouch Anastomosis Surgeries for IBD. J Crohns Colitis 10, 779-785. 10.1093/ecco-jcc/ jjv201.

Shogan, B.D., Belogortseva, N., Luong, P.M., Zaborin, A., Lax, S., Bethel, C., Ward, M., Muldoon, J.P., Singer, M., An, G., et al. (2015). Collagen degradation and MMP9 activation by Enterococcus faecalis contribute to intestinal anastomotic leak. Sci Transl Med 7, 286ra268. 10.1126/scitranslmed.3010658.

Shogan, B.D., Chen, J., Duchalais, E., Collins, D., Chang, M., Krull, K., Krezalek, M.A., Larson, D.W., WaltherAntonio, M.R., Chia, N., and Nelson, H. (2020). Alterations of the Rectal Microbiome Are Associated with the Development of Postoperative Ileus in Patients Undergoing Colorectal Surgery. J Gastrointest Surg 24, 16631672. $10.1007 / \mathrm{s} 11605-020-04593-8$.

van Praagh, J.B., de Goffau, M.C., Bakker, I.S., van Goor, H., Harmsen, H.J.M., Olinga, P., and Havenga, K. (2019). Mucus Microbiome of Anastomotic Tissue During Surgery Has Predictive Value for Colorectal Anastomotic Leakage. Ann Surg 269, 911-916. 10.1097/sla.0000000000002651.

van Praagh, J.B., de Wit, J.G., Olinga, P., de Haan, J.J., Nagengast, W.B., Fehrmann, R.S.N., and Havenga, K. (2021). Colorectal anastomotic leak: transcriptomic profile analysis. Br J Surg 108, 326-333. 10.1093/bjs/ znaa066. 
medRxiv preprint doi: https://doi.org/10.1101/2021.10.25.21265372; this version posted October 26, 2021. The copyright holder for this preprint (which was not certified by peer review) is the author/funder, who has granted medRxiv a license to display the preprint in It is made available under a CC-BY-NC-ND 4.0 International license.

Vandeputte, D., Falony, G., Vieira-Silva, S., Tito, R.Y., Joossens, M., and Raes, J. (2016). Stool consistency is strongly associated with gut microbiota richness and composition, enterotypes and bacterial growth rates. Gut 65, 57-62. 10.1136/gutjnl-2015-309618. 